\title{
Perturbation of Farthest Points in Weakly Compact Sets
}

\author{
Jean-Matthieu Augé
}

\begin{abstract}
If $f$ is a real valued weakly lower semi-continuous function on a Banach space $X$ and $C$ a weakly compact subset of $X$, we show that the set of $x \in X$ such that $z \mapsto\|x-z\|-f(z)$ attains its supremum on $C$ is dense in $X$. We also construct a counter example showing that the set of $x \in X$ such that $z \mapsto\|x-z\|+\|z\|$ attains its supremum on $C$ is not always dense in $X$.
\end{abstract}

\section{INTRODUCTION}

Throughout this paper, $X$ denotes a real Banach space, $B_{X}$ its closed unit ball, $X^{*}$ the Banach space of all continuous linear functionals on $X, C$ a bounded set of $X$ and $f: X \rightarrow \mathbb{R}$ a function which is bounded below on $C$. We study the following sets

$$
D(C, f)=\{x \in X ; \exists z \in C, r(x)=\|x-z\|-f(z)\},
$$

where by definition $r$ is the map from $X$ to $\mathbb{R}$ given by the formula

$$
r(x)=\sup \{\|x-z\|-f(z), z \in C\} .
$$

The map $r$ depends on $f$ and should be written $r_{f}$, but since there will be no ambiguity, we simply write $r=r_{f}$. We remark that $r$ is 1-Lipschitz and convex as a supremum of such functions and that by replacing $f$ by $f+a$ where $a$ is a constant, we can suppose that $f \geqslant 0$. When $f=0$, the set $D(C, 0)$ is geometrically the set of points of $X$ which admit a farthest point in the set $C$ and $r(x)$ is the farthest distance from $x$ to $C$, i.e. $r(x)$ is the smallest radius of the balls centered in $x$ that contain $C$. Here, the function $f$ is a perturbation, we will show that under suitable hypothesis of regularity on $f$, some results known on the set $D(C, 0)$ can be generalized. To be more precise, we will be interested in the generic existence of points in $D(C, f)$. For farthest points, the problem was first studied by Edelstein in [2] for uniformly convex spaces, assuming the set $C$ is bounded and norm closed and then generalized by Asplund in [1] for reflexive locally uniformly convex spaces. Then Lau in [4] showed that when $C$ is weakly compact (without any geometric hypothesis on $X$ ), the set of farthest points is dense and he

2000 Mathematics Subject Classification. Primary: 41A65.

Key words and phrases. Normed space, weakly compact set, farthest points. 
also showed that this result implies Asplund's theorem. Here we will give a generalization of Lau's theorem (see also the paper [5] which deals with Euclidean spaces, and [3] for the case of $p$-normed spaces): when $f$ is weakly lower semi-continuous and $C$ weakly compact, the set $D(C, f)$ contains a $G_{\delta}$ dense subset of $X$. We then take some particular $f$ to see what happens when we study the set of points $x \in X$ such that $z \mapsto\|z-x\|-\|z\|$ (resp. $z \mapsto\|z-x\|+\|z\|)$ attain their supremum on $C$.

\section{Density of the set $D(C, f)$}

We start this section by defining the sub-differential of the map $r$ (this definition stays unchanged for any convex map).

Definition 2.1. The sub-differential of $r$ is the set

$$
\partial r(x)=\left\{x^{*} \in X^{*} ; \forall y \in X,\left\langle x^{*}, y-x\right\rangle \leqslant r(y)-r(x)\right\} .
$$

Since $r$ is 1-Lipschitz, $\partial r(x)$ is contained in the closed unit ball of the dual. We can now state our positive theorem which follows the ideas of Lau's proof.

Theorem 2.1. Suppose that $C$ is a weakly compact subset of $X$ and that $f$ is weakly lower semi-continuous for the weak topology on $X$, then the set $D(C, f)$ contains a $G_{\delta}$ dense subset of $X$.

In order to prove the theorem, we will use the following lemma:

Lemma 2.1. Let $G=\left\{x \in X ; \forall x^{*} \in \partial r(x), \sup \left\{\left\langle x^{*}, x-z\right\rangle-f(z), z \in\right.\right.$ $C\}=r(x)\}$. Then $G$ is a $G_{\delta}$ dense subset of $X$.

Proof. Write $X \backslash G=\bigcup_{n=1}^{\infty} F_{n}$ with

$$
F_{n}=\left\{x \in X ; \exists x^{*} \in \partial r(x), \sup \left\{\left\langle x^{*}, x-z\right\rangle-f(z), z \in C\right\} \leqslant r(x)-\frac{1}{n}\right\} .
$$

By the Baire category theorem, it is enough to show that for fixed $n \geqslant 1$, $F_{n}$ is closed and nowhere dense.

- Let us first show that $F_{n}$ is a closed subset of $X$ : let $\left(x_{k}\right)$ be a sequence in $F_{n}$ converging to $x \in X$. By the definition of $F_{n}$, there exists $x_{k}^{*} \in \partial r\left(x_{k}\right)$ such that

$$
\forall z \in C, \forall k \geqslant 1,\left\langle x_{k}^{*}, x_{k}-z\right\rangle-f(z) \leqslant r\left(x_{k}\right)-\frac{1}{n} .
$$

Since $B_{X^{*}}$ is compact for $\sigma\left(X^{*}, X\right)$, we can choose $x^{*} \in \bigcap_{p}{\overline{\left\{x_{k}^{*}, k \geqslant p\right\}}}^{\sigma\left(X^{*}, X\right)}$, then we get for $z \in C$ :

$$
\begin{aligned}
\left|\left\langle x_{k}^{*}, x_{k}-z\right\rangle-\left\langle x^{*}, x-z\right\rangle\right| \leqslant & \left|\left\langle x_{k}^{*}, x_{k}-z\right\rangle-\left\langle x_{k}^{*}, x-z\right\rangle\right| \\
& +\left|\left\langle x_{k}^{*}, x-z\right\rangle-\left\langle x^{*}, x-z\right\rangle\right| \\
\leqslant & \left\|x_{k}^{*}\right\|\left\|x_{k}-x\right\|+\left|\left\langle x_{k}^{*}, x-z\right\rangle-\left\langle x^{*}, x-z\right\rangle\right| \\
\leqslant & \left\|x_{k}-x\right\|+\left|\left\langle x_{k}^{*}, x-z\right\rangle-\left\langle x^{*}, x-z\right\rangle\right| .
\end{aligned}
$$


Now for each fixed $z \in C$, there exists a subsequence $\left(x_{k_{q}}^{*}\right)$ such that $\left\langle x_{k_{q}}^{*}, x-\right.$ $z\rangle$ converges, and because $x^{*} \in \bigcap_{p}{\overline{\left\{x_{k}^{*}, k \geqslant p\right\}}}^{\sigma\left(X^{*}, X\right)}$, this limit is $\left\langle x^{*}, x-z\right\rangle$. By continuity of $r$, we obtain for each $z \in C$

$$
\left\langle x^{*}, x-z\right\rangle-f(z) \leqslant r(x)-\frac{1}{n},
$$

and hence

$$
\sup \left\{\left\langle x^{*}, x-z\right\rangle-f(z), z \in C\right\} \leqslant r(x)-\frac{1}{n} .
$$

To conclude that $x \in F_{n}$, it is enough to show that $x^{*} \in \partial r(x)$. Indeed, since $x_{k}^{*} \in \partial r\left(x_{k}\right)$, we have

$$
\forall y \in X,\left\langle x_{k}^{*}, y-x_{k}\right\rangle \leqslant r(y)-r\left(x_{k}\right)
$$

so by the same argument as before, we get at the limit: $x^{*} \in \partial r(x)$.

- Now, let us show that each $F_{n}$ is nowhere dense. Suppose it is false, then one can find $y_{0} \in X$ and $r>0$ such that $\bar{B}\left(y_{o}, r\right) \subset F_{n}$. Let $\alpha=$ $\sup \{\|z\|, z \in C\}, \lambda=\frac{r}{\alpha+\left\|y_{0}\right\|}$ and $\varepsilon=\frac{\lambda}{n(1+\lambda)}$. By the definition of $r\left(y_{0}\right)$, there exists $z_{0} \in C$ such that

$$
r\left(y_{0}\right)-\varepsilon<\left\|y_{0}-z_{0}\right\|-f\left(z_{0}\right) \leqslant r\left(y_{0}\right) .
$$

Finally, put $x_{0}=y_{0}+\lambda\left(y_{0}-z_{0}\right)$. With the choice of $\lambda$, we have $x_{0} \in$ $\bar{B}\left(y_{0}, r\right) \subset F_{n}$. Now, we estimate $r\left(y_{0}\right)-r\left(x_{0}\right)$ :

$$
r\left(y_{0}\right)-r\left(x_{0}\right)<\varepsilon+\left\|y_{0}-z_{0}\right\|-f\left(z_{0}\right)-r\left(x_{0}\right) \text {. }
$$

But,

$$
x_{0}=y_{0}+\lambda\left(y_{0}-z_{0}\right) \Longrightarrow x_{0}-z_{0}=(1+\lambda)\left(y_{0}-z_{0}\right) \text {. }
$$

Hence

$$
\begin{aligned}
r\left(y_{0}\right)-r\left(x_{0}\right) & <\varepsilon+\frac{1}{1+\lambda}\left\|x_{0}-z_{0}\right\|-f\left(z_{0}\right)-r\left(x_{0}\right) \\
& =\varepsilon+\frac{1}{1+\lambda}\left(\left\|x_{0}-z_{0}\right\|-f\left(z_{0}\right)\right)+\left(\frac{1}{1+\lambda}-1\right) f\left(z_{0}\right)-r\left(x_{0}\right) \\
& \leqslant \varepsilon+\frac{1}{1+\lambda} r\left(x_{0}\right)-\frac{\lambda}{1+\lambda} f\left(z_{0}\right)-r\left(x_{0}\right) \\
& =\varepsilon-\frac{\lambda}{1+\lambda} r\left(x_{0}\right)-\frac{\lambda}{1+\lambda} f\left(z_{0}\right) .
\end{aligned}
$$

Since $x_{0} \in F_{n}$, there exists $x^{*} \in \partial r\left(x_{0}\right)$ such that

$$
r\left(x_{0}\right) \geqslant \sup \left\{\left\langle x^{*}, x_{0}-z\right\rangle-f(z), z \in C\right\}+\frac{1}{n} \geqslant\left\langle x^{*}, x_{0}-z_{0}\right\rangle-f\left(z_{0}\right)+\frac{1}{n},
$$

which gives, combined with the last estimation:

$$
r\left(y_{0}\right)-r\left(x_{0}\right)<\varepsilon-\frac{\lambda}{1+\lambda}\left\langle x^{*}, x_{0}-z_{0}\right\rangle-\varepsilon=\left\langle x^{*}, y_{0}-x_{0}\right\rangle,
$$

which contradicts $x^{*} \in \partial r\left(x_{0}\right)$. 
Here, we have just used the fact that $C$ is bounded. The hypothesis of weak compactness of $C$ and of weak lower semi-continuity of $f$ allow us to finish the proof of the theorem as follows.

Proof. It is enough to see that $G \subset D(C, f)$. Consider $x \in G$ and $x^{*} \in \partial r(x)$, so

$$
\sup \left\{\left\langle x^{*}, x-z\right\rangle-f(z), z \in C\right\}=r(x) .
$$

Since $f$ is weakly lower semi-continuous and that $z \mapsto\left\langle x^{*}, x-z\right\rangle$ is weakly continuous, then $z \mapsto\left\langle x^{*}, x-z\right\rangle-f(z)$ is weakly upper semi-continuous on the weakly compact set $C$, and attains its supremum at a point $z_{0}$. We get:

$$
r(x) \leqslant\left\|x^{*}\right\|\left\|x-z_{0}\right\|-f\left(z_{0}\right) \leqslant r(x)
$$

because $\left\|x^{*}\right\| \leqslant 1$ and hence $r(x)=\left\|x-z_{0}\right\|-f\left(z_{0}\right)$.

Since $z \mapsto\|z\|$ is weakly lower semi-continuous, we obtain

Corollary 2.1. If $C$ is weakly compact, the set of $x \in X$ such that $z \mapsto$ $\|x-z\|-\|z\|$ attains its supremum on $C$ is dense in $X$.

\section{Counter examples And Remarks}

It is natural to ask ourselves if we can drop the hypothesis of weak lower semi-continuity in Theorem 2.1. The answer is no: more precisely, we construct the following counter example

Example 3.1. If $(K, d)$ is an infinite compact metric space and if $X=$ $C(K)$ is the space of real continuous functions on $K$ equiped with its usual norm, there exists a weakly compact subset $C$ of $X$ and a function $f$ weakly upper semi-continuous on $X$ such that $D(C, f)$ is not dense in $X$.

Indeed, take $f(z)=(1-\|z\|)^{+}=\max (0,1-\|z\|)$ and consider a decreasing sequence $\left(U_{n}\right)_{n \geqslant 1}$ of open subsets of $K$ such that $\bigcap_{n \geqslant 1} U_{n}=\emptyset$ (fix $y \in K$ which is not an isolated point in $K$, then a possible choice is $U_{n}=\{x \in$ $\left.\left.K \backslash\{y\} ; d(x, y)<\frac{1}{n}\right\}\right)$, let us also fix $t_{n} \in U_{n}$ and put

$$
x_{n}(t)=\frac{d\left(t, U_{n}^{c}\right)}{d\left(t, t_{n}\right)+d\left(t, U_{n}^{c}\right)} \quad(t \in K, n \geqslant 1) .
$$

By construction of $U_{n}$, we have $\left\|x_{n}\right\|=1$ and $\left(x_{n}\right)_{n \geqslant 1}$ converges pointwise to 0 which implies that $\left(x_{n}\right)_{n \geqslant 1}$ converges weakly to 0 as easily seen using the Riesz representation theorem and the Lebesgue's dominated convergence theorem. Put

$$
C=\left\{\left(1-\frac{1}{n}\right) x_{n}, n \geqslant 1\right\}=\{0\} \cup\left\{\left(1-\frac{1}{n}\right) x_{n}, n \geqslant 2\right\}
$$

which is weakly compact as the union of a convergent sequence and its limit. Note that $C$ is contained in $B_{X}$ and hence $f(z)=1-\|z\|$, we are left to find the supremum of the function $f_{x}(x \in X$ fixed) defined for $z \in C$ by $f_{x}(z)=\|x-z\|+\|z\|$. We will show that for $x \in \bar{B}(\mathbf{2}, 1)$ (where $\mathbf{2}$ denotes 
the function identically equal to 2$), f_{x}$ never attains its supremum and as a consequence $D(C, f)$ is not dense. Since for $t \in K, x(t) \geqslant 1$, we get for $z \in C$

$$
\|x-z\|=\sup |x(t)-z(t)|=\sup (x(t)-z(t)) \leqslant \sup x(t)=\|x\|
$$

and on the other hand $\|z\|<1$ gives $f_{x}(z)<\|x\|+1$. To finish, the last thing we have to see is that $\sup f_{x} \geqslant\|x\|+1$. Fix $t_{0}$ such that $\|x\|=\left|x\left(t_{0}\right)\right|$, then

$$
\sup f_{x} \geqslant f_{x}\left(\left(1-\frac{1}{n}\right) x_{n}\right) \geqslant\left|x\left(t_{0}\right)-\left(1-\frac{1}{n}\right) x_{n}\left(t_{0}\right)\right|+\left(1-\frac{1}{n}\right) .
$$

The conclusion follows because $\left(x_{n}\right)_{n \geqslant 1}$ converges pointwise to 0 .

Remark 3.1. - This last example also shows that the set of $x \in X$ such that $z \mapsto\|z-x\|+\|z\|$ attains its supremum on $C$ is not always dense in $X$. Recall that according to Corollary 2.1, the set of $x \in X$ such that $z \mapsto\|z-x\|-\|z\|$ attains its supremum on $C$ is always dense in $X$.

- There exists spaces, for example $l^{1}(\mathbb{N})$, or more generally any Banach space with the Schur's property where we can't construct any counter examples of the above type because the weakly and strongly compact sets coincide.

- However if $C=B_{X}$ and $X$ is reflexive (to ensure the weak compactness of $C$ ). The set of $x$ such that $f_{x}$ (defined by $f_{x}(z)=\|x-z\|+\|z\|$ ) attains its supremum on $C$ is dense. To show this, we use the following proposition.

Proposition 3.1. Let $f$ be a continuous convex function on $X, C$ a weakly compact subset of $X$ and $\varepsilon(C)$ the set of extremal points of $C$, then $\sup _{C} f=$ $\sup _{\varepsilon(C)} f$.

Proof. We have obviously, $\sup _{\varepsilon(C)} f \leqslant \sup _{C} f$. Suppose the reverse inequality is false and introduce $t$ such that

$$
\sup _{\varepsilon(C)} f<t<\sup _{C} f \text {. }
$$

Then, we have $\varepsilon(C) \subset C_{0}:=\{f \leqslant t\}$. Since $f$ is continuous convex, $C_{0}$ is a closed convex set, the Krein-Milman's theorem says that $\overline{\text { conv }}\|\cdot\|(\varepsilon(C))=C$, hence $C \subset C_{0}$. Now, since $\sup _{C} f>t$, one can find $x \in C$ such that $f(x)>t$ which contradicts $x \in C_{0}$.

This implies the last remark, indeed $\varepsilon(C)$ is of course contained in the unit sphere. Using the previous fact two times, we see that

$$
\sup _{z \in C} f_{x}(z)=\sup _{z \in \varepsilon(C)} f_{x}(z)=1+\sup _{z \in \varepsilon(C)}\|x-z\|=1+\sup _{z \in C}\|x-z\|
$$

which gives the conclusion with the main theorem (with the pertubation $f=0$ ). 
Remark 3.2. To finish, we would like to mention that the map $f \mapsto D(C, f)$ has no good properties. Let us take $X=\mathbb{R}, C=[0,1]$ and put for $z \in \mathbb{R}$, $f_{k}(z)=\frac{\mathbf{1}_{\{0,1\}}(z)}{k}$ where $\boldsymbol{1}_{\{0,1\}}$ denotes the characteristic function of the pair $\{0,1\}$ which is equal to 1 if $z=0$ or $z=1$ and 0 otherwise. It is obvious that $\left(f_{k}\right)_{k \geqslant 1}$ converges uniformly to $0(D(C, 0)=X)$ and yet, all the $D\left(C, f_{k}\right)$ are empty.

Indeed, let $x \in \mathbb{R}$ and suppose that $x \geqslant \frac{1}{2}$. For $z \in[0,1],|x-z|$ is maximal when $z=0$ and is equal to $x$. Hence

$$
\sup \left\{|x-z|-f_{k}(z), z \in[0,1]\right\} \leqslant x .
$$

On the other hand, taking a sequence $\left.\left(z_{n}\right) \subset\right] 0,1[$ converging to 0 , we get the reverse inequality. If we had a $z$ which attains the supremum, we should have

$$
f_{k}(z)=|x-z|-x \leqslant x-x=0,
$$

which implies that $z \in] 0,1[$. This gives us $|z-x|=x$ with $z \in] 0,1[$, which contradicts $|x-z|<x$. For $x \leqslant \frac{1}{2}$, we proceed the same way with the point $z=1$.

Acknowledgments. I would like to thank my advisor Robert Deville for his help during the elaboration of this paper as well as the referee for useful comments.

\title{
REFERENCES
}

[1] E. Asplund, Farthest points in reflexive locally uniformly rotund Banach spaces, Israel J. Math., 4 (1966), 213-216.

[2] M. Edelstein, Farthest points of sets in uniformly convex Banach spaces, Israel J. Math., 4 (1966), 171-176.

[3] S. Hejazian, A. Niknam, and S. Shadkam, Farthest Points and Subdifferential in pNormed Spaces, Hindawi Publishing Corporation International Journal of Mathematics and Mathematical Sciences, Volume 2008, Article ID 196326, 6 pages.

[4] K.S. Lau, Farthest points in weakly compact sets, Israel J. Math. 22 (1975), 168-174.

[5] X. Wang, On Chebyshev Functions and Klee Functions, submitted to Journal of Mathematical Analysis and Applications.

\author{
Jean-Matthieu Augé \\ Department of Mathematics \\ UNIVERSITY BORDEAUX 1 \\ 33400 TALENCE \\ FRANCE \\ E-mail address: jean-matthieu.auge@math.u-bordeaux1.fr
}

